\title{
The methodology of THz-VIS fused images evaluation
}

\author{
Marcin Kowalski, ${ }^{*}$ Norbert Palka, Marek Piszczek, Mieczyslaw Szustakowski \\ Institute of Optoelectronics, Military University of Technology, Kaliskiego 2, 00-908 Warszawa
}

Received March 18, 2013; accepted March 27, 2013; published March 31, 2013

\begin{abstract}
Image fusion can be used in a wide range of security applications, for example in detection and identification of hidden objects. Our goal is to build a harmless-to-humans system for screening and detecting hidden objects by means of a $\mathrm{THz}$ camera. A very important aspect of applying various processing techniques to images is the proper assessment of image quality. We propose a combination of two image quality assessment methods as a methodology of assessing the quality of fused images and a method to compare image fusion algorithms.
\end{abstract}

A growing interest in terahertz technology finds support in a large number of applications. One of the most interesting applications of terahertz waves is imaging. The terahertz range of electromagnetic radiation has large potential in the field of hidden objects detection because it is not harmful to humans. However, the main difficulty in the $\mathrm{THz}$ imaging systems is their low image quality due to low sensitivity and a small number of pixels in the detecting modules of cameras.

Considering the fact that even $\mathrm{THz}$ images with low pixel resolution still provide valuable information, it is justified to combine them with the high-resolution images from a visible camera [1]. Image fusion is a process of combining two or more images, or it can be defined as the process by which several images or parts of images (features) are combined into a single image. This process allows us to combine the most interesting elements of images from various spectral ranges into one image.

A very important aspect of applying various processing techniques to images is the proper assessment of image quality. Performance measures are essential to determine the possible benefits of fusion as well as to compare the results obtained with different algorithms [2]. During our investigations many different algorithms were implemented and tested. The Assessment of performance plays a crucial role in the process of choosing the best image fusion method.

The aim of our studies is to detect and visualize objects hidden under clothing. The detection of potentially dangerous objects is important, but without proper visualization, can be difficult to utilize. Security applications very often require a person to operate the system, thus a fused image is intended for presentation to a human observer for easier and enhanced interpretation.

\footnotetext{
*E-mail: mkowalski@wat.edu.pl
}

$\mathrm{THz}$ images are more difficult to assimilate for a human eye than visual images because they present a reality invisible for humans. Visible images are natural for human vision. Our studies show that the fused image should contain information mostly from the visible image with superimposed elements from the $\mathrm{THz}$ image showing a detected object.

In this paper we present the results of various image fusion methods and propose a method of assessing quality of the fused image by calculating the values of two image quality methods.

In our study image fusion is a way to enhance the visibility of selected features (objects hidden under clothing) in the observed scene.

Our image fusion methodology consists of three major steps. During the first step, the imaging material was acquired by two cameras - the THz and VIS. The $\mathrm{THz}$ images were acquired by a ThruVision TS4 camera operating at $250 \mathrm{GHz}$ with a pixel resolution of $124 \times 271$ points [3]. The camera working in the visible spectrum was manufactured by Axis and has a resolution of $600 \times 550$ pixels. Since the THz and VIS images have different fields of view and sizes we found a common area for both images [4]. This operation was the second step of the fusion process and is very often connected with geometrical transformations of images. In the second step the VIS image was subjected to a cropping operation while the $\mathrm{THz}$ image was thresholded. During the third step, one of selected image fusion method was applied to both, pre-processed images. The methodology of fusion process is shown in Fig. 1.

The following list of image fusion methods was used: ratio pyramid, gradient, shift invariant DWT (SIDWT), morphological pyramid, contrast pyramid, laplacian pyramid, averaging, principal component analysis (pca), minimum, maximum, discrete wavelet transform (dwt), and FSD pyramid [5-7].

The fusion algorithms may introduce distortions or artifacts into the processed images. So, image quality assessment (IQA) is essential to determine possible benefits of fusion as well as to compare results obtained with different algorithms. Furthermore, IQA methods are necessary in order to obtain an optimal setting of parameters for a specific fusion algorithm. All of the IQA methods can be divided into two groups - reference and 
no-reference methods. The reference methods use a reference image to measure the quality, while the noreference methods use only a resultant image to compute an image quality rate.

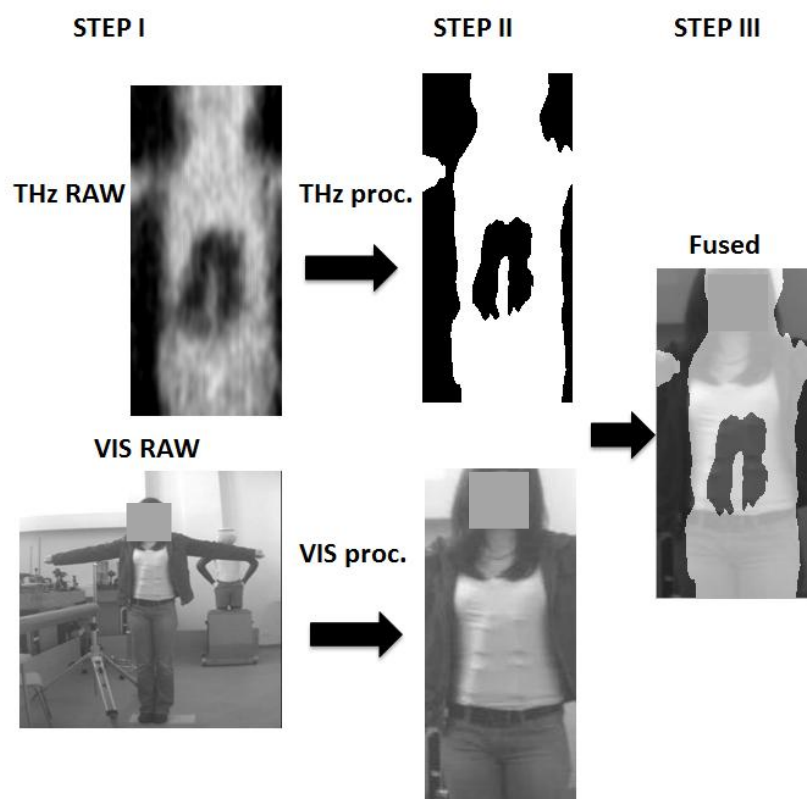

Fig. 1. Methodology of fusing images.

While the presence of a reference image or information regarding the reference simplifies the problem of quality assessment, the practical applications of such algorithms are limited in real-world scenarios where reference information is generally unavailable at nodes where quality computation is undertaken. The performance of any IQA method can be described by its correlation with human subjective judgments of quality, since the human is the "receiver" of the visual signal. Practical application of IQA methods requires that these algorithms compute a perceptual quality efficiently.

IQA in the case of a fused image is a complex task because after a process of image fusion one obtains an image that is an effect of transformation of two images ( $\mathrm{THz}$ and VIS) and it is not possible to find any reference image. A very popular method of evaluating the quality of fused images is calculating the values of the Peak Signal to Noise Ratio, Mean Squared Error or Average Difference [6, 7]. These methods work by exploiting the pixel differences between images, the correlation between images and changes in the histogram respectively. Our approach to assessing fused image quality is to evaluate the perception of images by a human eye. This was the reason why we used two kinds of IQA methods - the reference and the no-reference. We propose using the structural similarity index (SSIM) as the reference IQA method and the Natural Image Quality Evaluator (NIQE) as the no-reference (blind) quality assessment method. A combination of two unrelated image quality assessment methods can deliver the information required to assess the correctness of the fused image and select the most accurate fusion method.

The structural similarity (SSIM) index was invented for measuring the similarity between two images thus it is a full reference method [8]. The SSIM was designed to improve traditional methods which had seemed to be inconsistent with human eye perception. This method considers image degradation as perceived change in structural information. Structural information is the idea that the pixels have strong inter-dependencies especially when they are spatially close [8]. These dependencies carry important information about the structure of the objects in the visual scene. SSIM index values are in the range of $\langle 0,1\rangle$, where 1 is the value of two identical images. The SSIM index can be computed using the following equation [9]:

$$
\operatorname{SSIM}(x, y)=\frac{\left(2 \mu_{x} \mu_{y}+c_{1}\right)\left(2 \sigma_{x y}+c_{2}\right)}{\left(\mu_{x}^{2}+\mu_{x}^{2}+c_{1}\right)\left(\sigma_{x}^{2}+\sigma_{y}^{2}+c_{2}\right)},
$$

where $x, y$ are images, $\mu_{\mathrm{x}}\left(\mu_{\mathrm{y}}\right)$ is the average of $x(y), \sigma_{x}^{2}$ $\left(\sigma_{y}^{2}\right)$ is the variance of $x(\mathrm{y}), \sigma_{x y}$ is the covariance of $x$ and $y, c_{l}=\left(k_{1} L\right)^{2}, c_{2}=\left(k_{2} L\right)^{2}$ are two variables to stabilize the division with weak denominator, $L$ is the dynamic range of the pixel-values (typically $2^{\# \text { bits per pixel }}-1$ ), $k_{l}=0.01$ and $k_{2}=0.03$.

The second IQA method proposed in this paper is NIQE. It is an IQA method that makes use of measurable deviations from statistical regularities observed in natural images without training on human-rated distorted images, and without any exposure to distorted images.

NIQE is based on the construction of a quality aware collection of statistical features based on a simple and successful space domain natural scene statistic model. These features are derived from a corpus of natural, undistorted images [10].

The quality of the distorted image can be described as the distance between the natural scene statistic feature model and the multivariate Gaussian fit to the features extracted from the distorted image and can be computed using the following equation [10]:

$$
D\left(v_{1}, v_{2}, \Sigma_{1}, \Sigma_{2}\right)=\sqrt{\left(\left(v_{1}-v_{2}\right)^{T}\left(\frac{v_{1}+v_{2}}{2}\right)^{-1}\left(v_{1}-v_{2}\right)\right)}
$$

where $v_{1}, v_{2}$ are the mean vectors of the natural multivariate Gaussian model and the distorted image's multivariate Gaussian model, $\Sigma_{1}, \Sigma_{2}$ are the covariance matrices of the natural multivariate Gaussian model and the distorted image's multivariate Gaussian model. NIQE values are in the range of $<0, \infty$ ).

The results of applying image fusion methods to sample images (Fig. 1) as well as the results of IQA of fused images are presented in Table 1. 


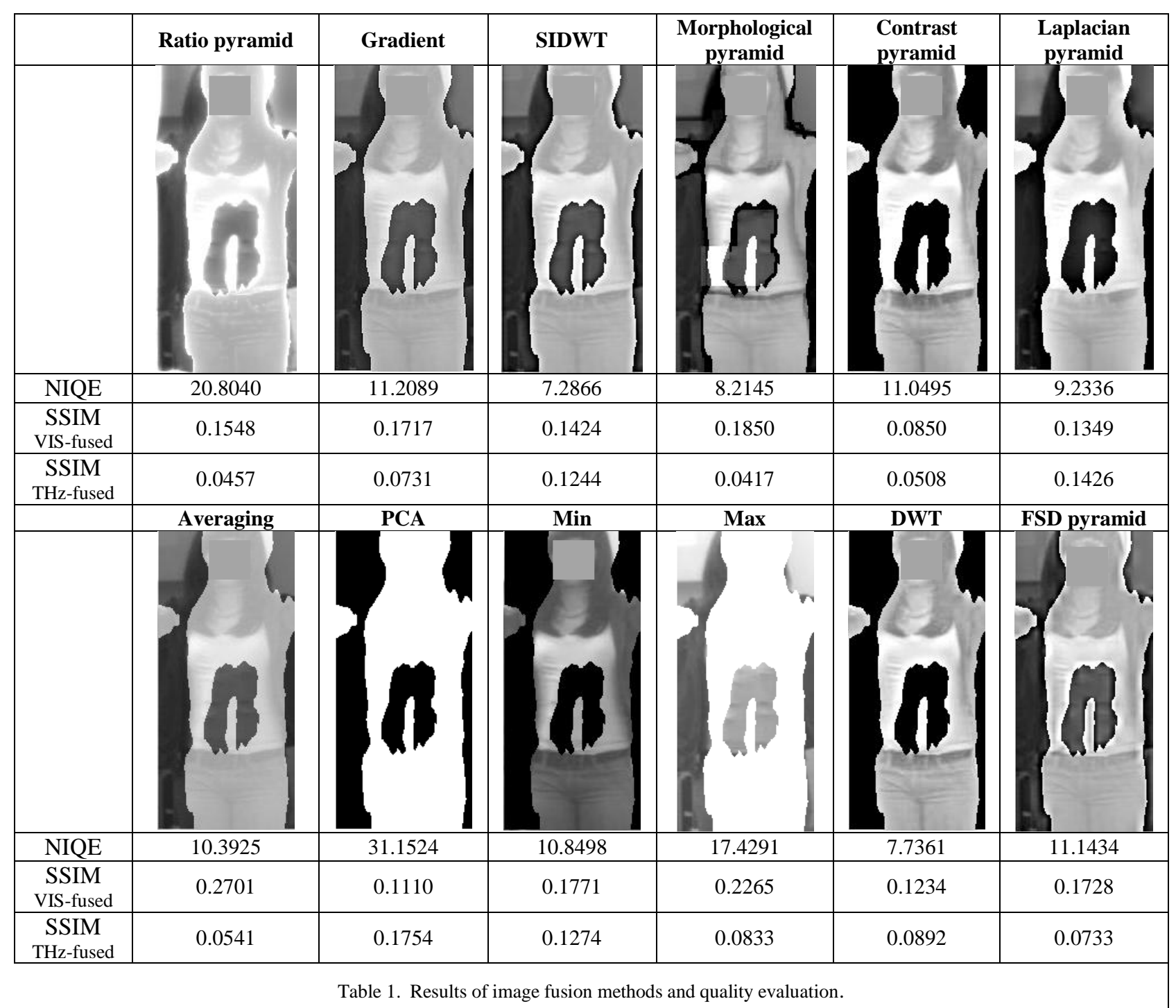

Based on the assumptions made at the beginning, we compared many series of the IQA results to select the best image fusion method.

According to our initial assumptions the value of the SSIM index of the VIS-fused images should tend to 1 while the value of SSIM index of the THz-fused images and the value of NIQE should tend to 0 . This means that the correlation index between the fused and the VIS images is bigger than between the fused and the $\mathrm{THz}$ ones. It is natural because the VIS image is the background image for the fusion process, and only a few necessary and relevant elements of $\mathrm{THz}$ image are used. After analysis of many series of results the averaging method was chosen. The selected method was modified by replacing average with weighted average.

The methodology of evaluating image quality presented in the paper shows that it is possible to assess the quality of images and human perception of images by using a combination of various IQA methods. This methodology was very useful during investigations into image fusion techniques and it allowed us to choose the most appropriate fusion algorithm for further system implementation.

\section{References}

[1] V.A. Trofimov et al., Proc. SPIE 8189 (2011).

[2] M. Piszczek, M. Kowalski, M. Szustakowski, Acta Phys. Pol. A 120, 720 (2011)

[3] ThruVision System Ltd. website: http://www.thruvision.com.

[4] C. Toxqui-Quitl et al., Proc. SPIE 5558 (2004).

[5] M. Kowalski, M. Piszczek, N. Palka, M. Szustakowski, Phot. Lett. Poland 4, 3 (2012)

[6] N.N. Wang, J.H. Qiu, W.B. Deng, Infrared Techn. 31, N3, 129 (2009).

[7] M. Sumathi, R. Barani, Proc. of IEEE, PRIME (2012).

[8] W. Pei, G. Wang, X. Yu, Proc. of IEEE, IGARSS (2012).

[9] D. Brunet, E.R. Vrscay, Z. Wang, IEEE Trans. on Image Proc. 21, 4 (2012).

[10] Z. Wang, A. Bovik, IEEE Trans. on Image Proc 13, 4 (2004).

[11] A. Mittal, R. Soundararajan, A. C. Bovik, IEEE Sig. Proc. Lett., 20 (2013). 